Gebhardt, Michael; Rudolph, Tobias; Kampke, Wolfgang; Eisenhauer, Norbert Fischabstieg über Schlauchwehre: Untersuchungen der Strömungsverhältnisse und Identifizierung der Abflussbereiche mit erhöhtem Verletzungsrisiko

Verfügbar unter / Available at:

https://hdl.handle.net/20.500.11970/100672

Vorgeschlagene Zitierweise / Suggested citation:

Gebhardt, Michael; Rudolph, Tobias; Kampke, Wolfgang; Eisenhauer, Norbert (2014): Fischabstieg über Schlauchwehre: Untersuchungen der Strömungsverhältnisse und Identifizierung der Abflussbereiche mit erhöhtem Verletzungsrisiko. In: Wasserwirtschaft Jg. 104 (7-8/2014). S. 48-53. 
Erstveröffentlichung in WasserWirtschaft 7/8(2014), S. 48-53.

Für eine korrekte Zitierbarkeit ist die Seitennummerierung der Originalveröffentlichung für jede Seite kenntlich gemacht.

\section{Fischabstieg über Schlauchwehre: Untersuchungen der Strö- mungsverhältnisse und Identifizierung der Abflussbereiche mit erhöhtem Verletzungsrisiko}

Michael Gebhardt, Tobias Rudolph, Wolfgang Kampke und Norbert Eisenhauer

Wie nahezu alle überströmten Wehrverschlüsse können Schlauchwehre an Standorten mit niedrigem Unterwasserstand aus ökologischer Sicht problematisch sein, weil dann die Gefahr besteht, dass sich abwandernde Fischean der Wehrschwelle verletzen. Mit Hilfe numerischer und hydraulischer Untersuchungen werden diese Abflussbereiche identifiziert und die relevanten hydraulischen Größen quantifiziert. Des Weiteren werden bauliche und betriebliche Maßnahmen untersucht und bewertet, die die Verletzungsgefahr von Fischen beim Abstieg über Schlauchwehre auf ein Minimum reduzieren.

\section{Motivation}

Beim stromabwärts gerichteten Wandern orientieren sich Fische nach derzeitigem Kenntnisstand an der Hauptströmung und nutzen daher je nach Abfluss Wasserkraftanlage, Wehr oder Fischabstiegshilfen, sofern sie vorhanden sind. Während die Passage über das Wehr im Vergleich zur Turbinenpassage sicher erscheint, gibt es auch hier eine Reihe von Gefährdungsfaktoren, die vom Verschlusstyp, der Über- und Unterströmung, dem Vorhandensein von Tosbeckeneinbauten und der Entwicklung der Unterwasserstände abhängen.

Wie bei allen Verschlusstypen wird auch beim Schlauchwehr versucht, die Verschlusshöhe durch eine Jambor-Schwelle zu reduzieren [5]. Je nach Unterwasserstand und Abfluss gibt es dabei Zustände, bei denen der Überfallstrahl aufdie Wehrschwelle bzw. den Ablagetisch mit einemgeringen Wasserpolster fällt (Bild 1, links), woraus u. U. ein erhöhtes Verletzungsrisiko für absteigende Fische entsteht. Ziel ist es, die Überströmung von Schlauchwehren qualitativ und quantitativ zu charakterisieren, um damit die Schädigungsgefahr für Fische besser einschätzen zu können.

\section{Der Fischabstieg über Wehranlagen}

Beim Abstieg können sowohl aktive als auch passive Bewegungen (Schwimmen mit bzw. gegen die Strömung) beobachtet werden. Hinsichtlich der bevorzugten Wassertiefe sind artspezifische Unterschiede feststellbar. Beispielsweise wandern Lachssmolts und die Jungfische vieler Weißfischarten oberflächennah stromabwärts, während Aale und Neunaugen sohlennahe Bereiche bevorzugen (z. 


\section{Autorenfassung}

Gebhardt, Rudolph, Kampke, Eisenhauer: Fischabstieg über Schlauchwehre: Untersuchungen der Strömungsverhältnisse und Identifizierung der Abflussbereiche mit erhöhtem Verletzungsrisiko, 2014

B. [1], [2]). Allen Arten gemein sind offenbar eine Orientierung an der Hauptströmung und ein gehäuftes Auftreten in Bereichen hoher Strömungsenergie bzw. des Stromstrichs [2]. Bei einer Abflussmenge bis zur Ausbauwassermenge des Kraftwerks wird der Fisch daher die Tendenz haben, vor das Kraftwerk zu gelangen.

Untersuchungen zur Mortalität bei der Turbinenpassage weisen eine erhebliche Spanne auf: Bei Francis-Turbinen in Laufwasserkraftwerken wurden beispielsweise für juvenile Salmoniden Mortalitätsraten von weniger als $5 \%$ bis über $90 \%$ und bei Kaplan-Turbinen von $5 \%$ bis $20 \%$ ermittelt [1]. Im Allgemeinen kann davon ausgegangen werden, dass die Mortalitätsra-
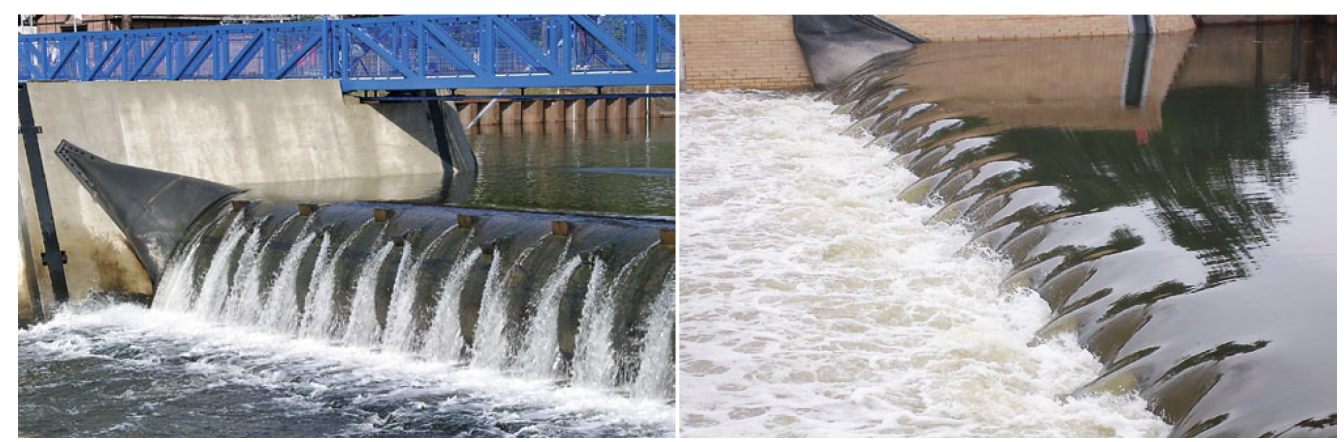

Bild 1: Überströmtes Schlauchwehr mit geringem (links) und erhöhtem Unterwasserstand (rechts)

Gebhardt, Rudolph, Kampke, Eisenhauer: Fischabstieg über Schlauchwehre: Untersuchungen der Strömungsverhältnisse und Identifizierung der Abflussbereiche mit erhöhtem Verletzungsrisiko: Titel des Artikels. WasserWirtschaft $7 / 8$ (2014), S. 48-53.

te der Fische bei der Passage über das Wehr niedriger ist als bei der Turbinenpassage und daher die Mortalitätsrate einer Staustufe in den meisten Fällen vom Kraftwerk dominiert wird. Dies zeigen verschiedene Untersuchungen, wie beispielsweise von Muir et al. [8] für Königslachse und Regenbogenforellen im Snake River (USA), aber auch eigene Untersuchungen auf Basis wahrscheinlichkeitstheoretischer Betrachtungen [12].

Zum Abstieg über Wehranlagen liegen bislang hauptsächlich Untersuchungsergebnisse aus Nordamerika zu Schädigungen an Lachsen verschiedener Arten und Entwicklungsstadien vor. Bei der Mehrzahl der in den Studien aufgeführten Fallbeispiele liegen die Mortalitätsraten in einer ähnlichen Größenordnung 3 bis $9 \%$ [9] bzw. 0 bis 7 \% [6], in Einzelfällen wurden aber auch Mortalitätsraten von $37 \%$ ermittelt [11]. Zu diesen Untersuchungen ist anzumerken, dass die Stauanlagen zumeist mit unterströmten Verschlüssen, Schützen und Drucksegmenten ausgestattet sind und diese Fallhöhen hauptsächlich zwischen 15,0 $\mathrm{m}$ und 30,0 m, aber auch bis zu 75,0 $\mathrm{m}$ aufweisen. 


\section{Autorenfassung}

Gebhardt, Rudolph, Kampke, Eisenhauer: Fischabstieg über Schlauchwehre: Untersuchungen der Strömungsverhältnisse und Identifizierung der Abflussbereiche mit erhöhtem Verletzungsrisiko, 2014

Die DWA [1] stellt fest, dass Schädigungen bei überströmten Verschlüssen und Fallhöhen unter 13,0 m gering ausfallen dürften, wenn die Unterwassertiefe etwa ein Viertel der Fallhöhe, mindestens aber 0,9 m, beträgt. Bei der Passage von unterströmten Verschlüssen wird hingegen eine höhere Mortalitätsrate erwartet, die auf die plötzlichen Druckänderungen bzw. die hohen Geschwindigkeitsgradienten zurückzuführen ist.

\section{Methodik}

\subsection{Numerische Modelluntersuchungen}

Die Berechnungen mit der Software OpenFOAM wurden zur Minimierung des Rechenaufwandes an einem Ausschnittsmodell durchgeführt, dessen Abmessungen in der Länge einem bestehenden Labormodell entsprachen. Aus Symmetriegründen wurde in der Breite ein Abschnitt zwischen der jeweiligen Achse von zwei Störkörpern betrachtet. Da die Störkörper bei kleinen Abflüssen und der daraus resultierenden geringen Überfallhöhe einen großen Einfluss auf das Strömungsbild haben, wurde der Nahbereich der Störkörper entsprechend höher aufgelöst. Eine höhere Auflösung erfolgte auch im Bereich der zu erwartenden Wasserspiegeloberfläche, um die Luft-Wasser-Grenze scharf abzubilden. Somit ergab sich für das 6,60 m lange und 0,13 m breite Modell in Abhängigkeit des untersuchten Zustandes ein Berechnungsgitter mit 1,7 Mio. bis 2,0 Mio. Zellen (Bild 2).

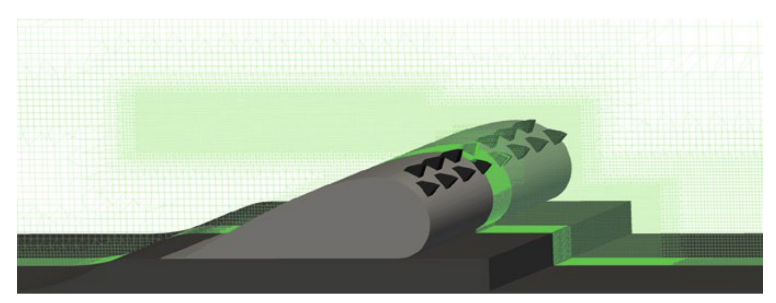

Bild 2: Ausschnittmodell eines Schlauchwehres mit räumlich diskretisiertem Berechnungsgitter 


\section{Autorenfassung}

Gebhardt, Rudolph, Kampke, Eisenhauer: Fischabstieg über Schlauchwehre: Untersuchungen der Strömungsverhältnisse und Identifizierung der Abflussbereiche mit erhöhtem Verletzungsrisiko, 2014

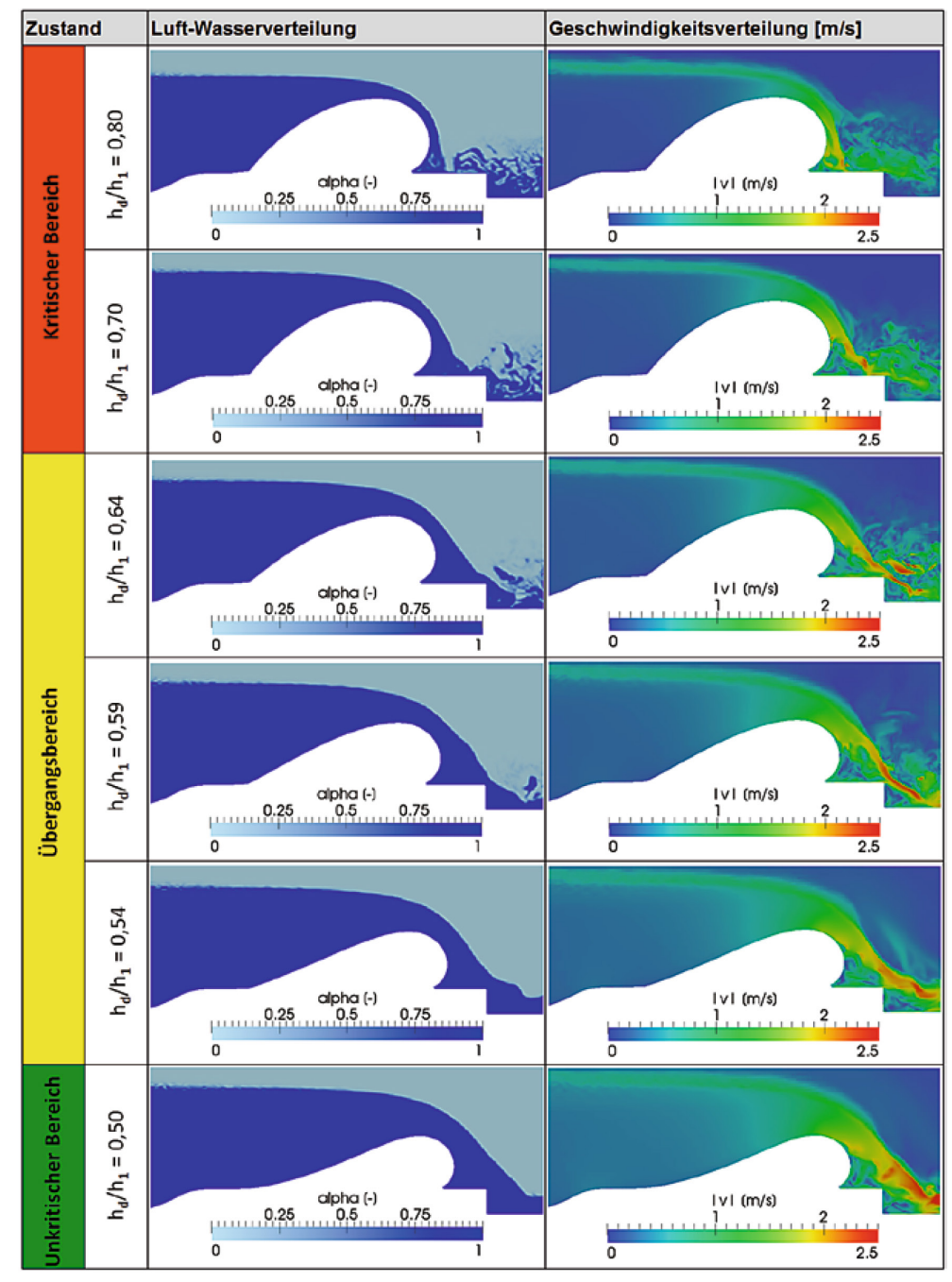

Bild 3: Simulationsergebnisse für die Abflusszustände $h_{d} / h_{1}=0,80$ bis $h_{d} / h_{1}=0,50$ : LuftWasser-Verteilung (links) und Geschwindigkeitsverteilung in Luft und Wasser (rechts)

Gebhardt, Rudolph, Kampke, Eisenhauer: Fischabstieg über Schlauchwehre: Untersuchungen der Strömungsverhältnisse und Identifizierung der Abflussbereiche mit erhöhtem Verletzungsrisiko: Titel des Artikels. WasserWirtschaft 7/8 (2014), S. 48-53.

\subsection{Bestimmung der Schlauchgeometrie}

Der Querschnitt eines Schlauchwehres und die Schlauchhöhe $h_{d}$ ändern sich über das Abflussspektrum in Abhängigkeit von Innendruck, Ober- und Unterwasserstand. Da die Schlauchgeometrie für die Berechnung eine feste Strömungsberandung darstellt, muss diese für die numerisch zu untersuchenden Zustände bekannt sein. Zwar lag die Geometrie aus Laboruntersuchungen vor, musste allerdings auf der Luftseite ergänzt werden, da mit der verwendeten Messtechnik nicht die voll- 


\section{Autorenfassung}

Gebhardt, Rudolph, Kampke, Eisenhauer: Fischabstieg über Schlauchwehre: Untersuchungen der Strömungsverhältnisse und Identifizierung der Abflussbereiche mit erhöhtem Verletzungsrisiko, 2014

ständige Oberfläche erfasst werden konnte. Gebhardt [4] beschreibt ein analytisches Verfahren zur Bestimmungder Schlauchgeometrie, das verwendet wurde, um die Geometrie zu vervollständigen.

Für die numerischen Modelluntersuchungen ergeben sich somit zwölf verschiedene Geometrien mit Schlauchhöhen $h_{d}$ von 0,08 bis 0,22 m. Die Schwellenhöhe w beträgt konstant 0,06 m, wobei sich die Wehrschwelle aus der Jambor-Schwelle und einem Ablagetisch zusammensetzt, dessen Länge der abgelegten Membran zzgl. einem Arbeitsraum von etwa 0,06 m entspricht. Diese Ausgangsgeometrie wird im Folgenden als „Standard-Ablagetisch“ bezeichnet.

\subsection{Untersuchte Abflusszustände}

Für die zu untersuchenden Abflusszustände wurde ein konstanter Unterwasserstand $\mathrm{h}_{2}$ gewählt, der unterhalb der Oberkante der Wehrschwelle liegt $\left(\mathrm{h}_{2}<\mathrm{w}\right)$ und damit die Empfehlungen der DWA [1] deutlich unterschreitet. Der Abfluss Q wurde bei konstantem Oberwasserstand $\mathrm{h}_{1}=0,26 \mathrm{~m}$ von 0,025 bis $0,300 \mathrm{~m}^{3} / \mathrm{s}$ variiert, so dass sich insgesamt zwölf Abflusszustände mit dimensionslosen Schlauchhöhen zwischen $h_{d} / h_{1}=0,29$ und $h_{d} / h_{1}=0,86$ ergaben.

\section{Ergebnisse mit dem Standard-Ablagetisch}

\subsection{Charakteristik des Wehrüberfalls}

In einem ersten Schritt wurde der Wehrüberfall in Abhängigkeit des Abflusses charakterisiert. Hierbei lag der Fokus auf Auftreffpunkt, -winkel und -geschwindigkeit des Überfallstrahls, wobei jeweils die Strahlunterseite betrachtet wurde. In Bild 3 sind die Simulationsergebnisse für sechs Abflusszustände repräsentativ für drei unterschiedlicheBereiche gegenübergestellt. Dargestellt ist die Luft-Wasser-Verteilung(Bild 3, links), wobei alpha $=0$ (Luft) und alpha $=1$ (Wasser) bedeutet. In Bild 3 (rechts) ist die Geschwindigkeitsverteilung in Luft und Wasser zu erkennen. In Bild 4 sind zum Vergleich drei ausgewählte Zustände im Labormodell dargestellt.

Unter den zwölf Abflusszuständen konnten somit drei Bereiche identifiziert werden:

\section{Kritischer Bereich}

Bis $h_{d} / h_{1}=0,80$ trifft der Überfallstrahl vollständig und mit steilem Winkel auf die Wehrschwelle. Aufgrund der Ablenkung an den Störkörpern liegt der Auftreffpunkt der zweiten Störkörperreihe weiter unterstrom mit einer Art Wechselsprung auf bzw. im Anschluss an die Wehrschwelle. Im Gegensatz dazu erfährt der Strahlanteil hinter der ersten Reihe eine Ablenkung schräg nach oben, wobei durch die Strahlversprühung ein hoher Lufteintrag zu beobachten ist. Ein kleiner Abflussanteil, nach rückwärts auf das Schlauchwehr gerichtet, führt hinter dem Überfallstrahl zu einem Rückstau und einem Wasserstand, der höher ist als im Unterwasser. Mit steigendem Abfluss bildet sich unterhalb des Überfallstrahls eine Walze aus, und der Auftreffwinkel wird flacher. $\mathrm{Ab} \mathrm{h}_{\mathrm{d}} / \mathrm{h}_{1}=0,70$ ist zu beobachten, dass der Strahl abgelenkt wird, und horizontal über die Wehrschwelle hinausschießt. 


\section{Autorenfassung}

Gebhardt, Rudolph, Kampke, Eisenhauer: Fischabstieg über Schlauchwehre: Untersuchungen der Strömungsverhältnisse und Identifizierung der Abflussbereiche mit erhöhtem Verletzungsrisiko, 2014

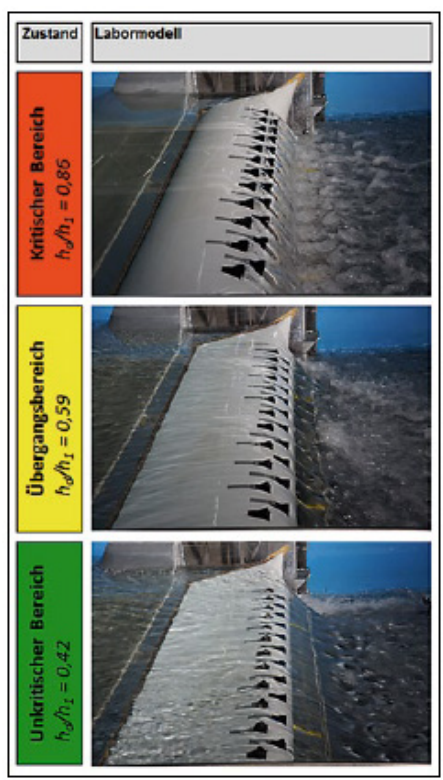

Bild 4: $\quad$ Abflusszustände $h_{d} / h_{1}=0,86, h_{d} / h_{1}=0,59$ und $h_{d} / h_{1}=0,42$ im Labormodell

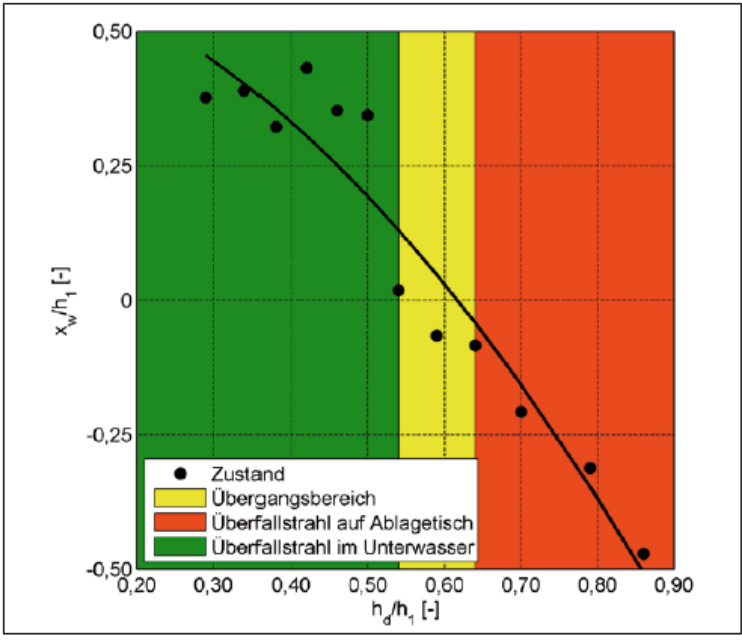

Bild 5: $\quad$ Charakterisierung des Überfallstrahls bei einem Schlauchwehr mit StandardAblagetisch in Abhängigkeit vom Auftreffpunkt des Überfallstrahls 


\section{Autorenfassung}

Gebhardt, Rudolph, Kampke, Eisenhauer: Fischabstieg über Schlauchwehre: Untersuchungen der Strömungsverhältnisse und Identifizierung der Abflussbereiche mit erhöhtem Verletzungsrisiko, 2014

Gebhardt, Rudolph, Kampke, Eisenhauer: Fischabstieg über Schlauchwehre: Untersuchungen der Strömungsverhältnisse und Identifizierung der Abflussbereiche mit erhöhtem Verletzungsrisiko: Titel des Artikels. WasserWirtschaft 7/8 (2014), S. 48-53.

\section{Übergangsbereich}

Der Auftreffpunkt des Überfalllstrahls erreicht das Ende der Wehrschwelle und der Strahl fällt in großen Teilen ohne Umlenkung ins Unterwasser. Der Lufteintrag auf der Wehrschwelle ist deutlich niedriger. $A b h_{d} / h_{1}=0,59$ ist zu beobachten, dass der Strahl die Tosbeckensohle erreicht und dort die größten Fließgeschwindigkeiten auftreten. Im Tosbecken bildet sich ein Wechselsprung mit einer Deckwalze aus.

\section{Unkritischer Bereich}

$\mathrm{Ab} \mathrm{h}_{\mathrm{d}} / \mathrm{h}_{1}=0,46$ fällt der Überfallstrahl in voller Strahlstärke und mit flachem Auftreffwinkel ins Unterwasser. Zwischen Strahl und Wehrschwelle bildet sich eine Walze aus, die aber mit weiter abgelegtem Schlauch immer kleiner wird. Über der Wehrschwelle liegt schießender Abfluss vor und der Strahl ist praktisch unbeeinflusst von der Wehrschwelle.

Bild 5 zeigt die Auswertung für den Auftreffpunkt des Überfallstrahls im untersuchten Abflussspektrum ( $\mathrm{x}_{\mathrm{w}}$ bezeichnet den horizontalen Abstand zum Schwellenende). Für $\mathrm{x}_{\mathrm{w}} / \mathrm{h}_{1}<0$ fällt der Überfallstrahlauf den Ablagetisch (rot) und für $\mathrm{x}_{\mathrm{w}} / \mathrm{h}_{1}>0$ ins Unterwasser (grün). Im Übergangsbereich (gelb) fällt der Strahl zwar ebenfalls in großen Teilen ins Unterwasser, die Strahlgeometrie wird aber noch vom Schwellenende beeinflusst. Hier zeigt sich, dass die Abflusszustände mit einer Schlauchhöhe unter $h_{d} / h_{1}=0,54$ unbeeinflusstvon der Wehrschwelle sind und daher als unkritisch für den Fischabstieg angesehen werden. Der Überfallstrahl fällt direkt ins Unterwasser und ein Kontakt des abwandernden Fischs mit der Wehrschwelle ist nicht zu erwarten. Im Übergangsbereich ist ebenfalls von einem geringen Verletzungsrisiko auszugehen. Für Schlau chhöhengrößer als $\mathrm{h}_{\mathrm{d}} / \mathrm{h}_{1}=0,64$ trifft der Überfallstrahl hingegen auf den Ablagetisch und durchschlägt das geringe Wasserpolster. In diesem kritischen Bereich sind äußere und innere Verletzungen bei Fischen durch den Kontakt mit der Wehrschwelle oder durch die Gasblasenkrankheit infolge des starken Lufteintrages nicht auszuschließen.

\subsection{Druckänderungen und Geschwindigkeitsgradienten im Überfallstrahl}

Nach DWA [1] kann eine erhöhte Mortalität auch infolge plötzlicher Druckschwankungen bzw. Änderungen der Strömungsgeschwindigkeit auftreten. Daher wurden in einem weiteren Schritt die Strömungsgrößen bestimmt, die ein Fisch bei der Passage im Überfallstrahl erfährt. Hierzu wurde ein Abstiegsweg in der Mitte des Überfallstrahls angenommen sowie Fließgeschwindigkeit und Druck entlang diskreter Punkte bestimmt. In Bild 6a ist die Fließgeschwindigkeit für die sechs Abflusszustände dargestellt, in Bild 6b der entsprechende Druckverlauf. Für die beiden Zustände ist 


\section{Autorenfassung}

Gebhardt, Rudolph, Kampke, Eisenhauer: Fischabstieg über Schlauchwehre: Untersuchungen der Strömungsverhältnisse und Identifizierung der Abflussbereiche mit erhöhtem Verletzungsrisiko, 2014

im kritischen Bereich $\left(h_{d} / h_{1}=0,80\right.$ bzw. $\left.h_{d} / h_{1}=0,70\right)$ zu erkennen, dass die Geschwindigkeit - wie zu erwarten - vom Scheitelpunkt bis zur Wehrschwelle auf bis zu 1,65 m/s zunimmt und dort, wo der Überfallstrahl auftrifft, plötzlich abnimmt. Hierbei ist auch ein Druckanstieg festzustellen. Beim Überfall von der Wehrschwelle ins Unterwasser wird die Strömung anschließend wieder beschleunigt. Die negative Beschleunigung, die der Überfallstrahl und damit der Fisch beim Auftreffen auf die Wehrschwelle erfährt, wird mit steigendem Abfluss kleiner $\left(h_{d} / h_{1}=0,64\right.$ und $\left.h_{d} / h_{1}=0,54\right)$ und die Geschwindigkeit steigtkontinuierlich bis ins Unterwasser an, da der Überfallstrahl nicht mehr von der Wehrschwelle abgelenkt wird: Ein absteigender Fisch erfährt damit eine kontinuierliche Beschleunigung.

Zur Beurteilung von Druckänderungen wurde der zeitliche Verlauf aus Fließgeschwindigkeit und Druck entlang des Abstiegsweges berechnet (Bild 6c). Die Druckänderung $\Delta \mathrm{P}$ ergibt sich dabei aus der Druckdifferenz, der mittleren Fließgeschwindigkeitund dem Abstand von zwei aufeinanderfolgenden, örtlich diskreten Berechnungsergebnissen. Im Vergleich der sechs Zustände ist zu beobachten, dass die Druckänderung negativ ist und vom Scheitel kontinuierlich abnimmt. Im Bereich der Störkörper steigt der Druck um etwa 125 Pa und beim Auftreffen auf die Wehrschwelle um weitere etwa 500 Pa innerhalbvon $0,02 \mathrm{~s}$ an $\left(\mathrm{h}_{\mathrm{d}} / \mathrm{h}_{1}=0,70\right)$. Die Druckänderungen verringern sich mit steigendem Abfluss.

In Bild 6d ist die sich aus der Änderung der Fließgeschwindigkeit ergebende Beschleunigung dargestellt. Dabei ist zu erkennen, dass der Fisch beim Abflusszustand $h_{d} / h_{1}=0,80$ eine gleichmäßig beschleunigte Bewegung erfahren würde, die etwa bei $t=0,30$ s ein Maximum von über 2 g erreicht. Dies entspricht dem Auftreffpunkt des Überfallsstrahls auf die Wehrschwelle. Durch das Auftreffen tritt

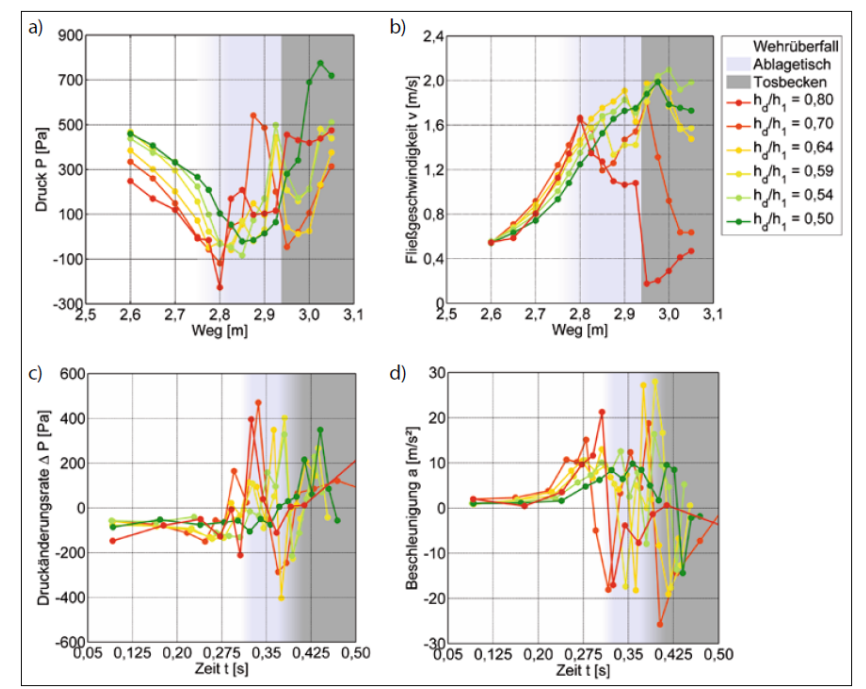

Bild 6: $\quad$ a) Druck, b) Fließgeschwindigkeit, c) Druckänderungund d) Beschleunigungin der Mitte des Überfallstrahls für die Zustände $h_{d} / h_{1}=0,80$ bis $h_{d} / h_{1}=0,50$ (Referenz: Atmosphärendruck) 


\section{Autorenfassung}

Gebhardt, Rudolph, Kampke, Eisenhauer: Fischabstieg über Schlauchwehre: Untersuchungen der Strömungsverhältnisse und Identifizierung der Abflussbereiche mit erhöhtem Verletzungsrisiko, 2014

Gebhardt, Rudolph, Kampke, Eisenhauer: Fischabstieg über Schlauchwehre: Untersuchungen der Strömungsverhältnisse und Identifizierung der Abflussbereiche mit erhöhtem Verletzungsrisiko: Titel des Artikels. WasserWirtschaft 7/8 (2014), S. 48-53.

dann eine negative Beschleunigung von etwa $2 \mathrm{~g}$ auf.

\section{3 Übertragung vom Modell in die Natur}

Mit Hilfe der Froude‘schen Ähnlichkeit können die oben angegebenen Größen auf eine beliebige geometrisch ähnliche Anlage in der Natur umgerechnet werden. In Tabelle 1 sind die maßgeblichen Strömungsparameter für vier ausgewählteVerschlusshöhen zwischen 2,0 m und 5,0 m angegeben. Aus dem spezifischen Abfluss q kann der Gesamtabfluss für beliebige Wehrfeldbreiten ermittelt werden.

\section{Ergebnisse modifizierter Wehrschwellen}

\subsection{Untersuchte Schwellengeometrien}

Die bisherigen Ergebnisse zeigen, dass die größte Verletzungsgefahr für Fische durch den Kontakt mit der Wehrschwelle entsteht. Aus diesem Grund wurden modifizierte Schwellengeometrien untersucht (Bild 7) und deren Einfluss auf die Charakteristik des Wehrüberfalls analysiert. Neben einer Abschrägung bzw. Ausrundung des Schwellenendes wurde auch eine Aufkantung auf dem Ablagetisch betrachtet mit dem Ziel, bei kleinen Abflüssen ein Wasserpolster zu generieren. Aufgrund der hydraulischen Nachteile (reduzierte Abflussleistung, Schwingungsgefahr) wurde diese Möglichkeit aber nicht weiter verfolgt. Die beiden ersten Varianten ergaben keine nennenswerten Verbesserungen, da der Überfallstrahl nach wie vor auf den Ablagetisch fiel [10].

\subsection{Ausführungsvorschlag}

Im günstigsten Fall kann der Ablagetisch um die Breite des Arbeitsraumes verkürzt werden. Die Berechnungsergebnisse für diese Variante zeigen, dass durch die Verkürzung der kritische Bereich von $h_{d} / h_{1} \geq 0,64$ auf $h_{d} / h_{1} \geq 0,70$ verkleinert werden kann. Das bedeutet, der Überfallstrahl fällt bereits bei kleineren Abflüssen direkt ins Unterwasser.

Neben der Verkürzung des Ablagetisches kann der Übergang in das Tosbecken durch eine Rampe erfolgen. Diese Neigung ist zwar hydrodynamisch nicht zwingend erforderlich, da sich in diesem Bereich ohnehin eine Ablösezone bildet. Allerdings rotiert dort auch mitgeführtes Geschiebe und führt u. U. zu Schäden an der Betonoberfläche. Im vorliegenden Fall wird eine Neigung von $45^{\circ}$ vorgeschlagen, was in etwa dem Auftreffwinkel des Überfallstrahls entspricht. 


\section{Autorenfassung}

Gebhardt, Rudolph, Kampke, Eisenhauer: Fischabstieg über Schlauchwehre: Untersuchungen der Strömungsverhältnisse und Identifizierung der Abflussbereiche mit erhöhtem Verletzungsrisiko, 2014

Die Ecken sind mit $\mathrm{R}_{1} / \mathrm{w}=0,40$ bzw. $\mathrm{R}_{2} / \mathrm{w}=0,40$ ausgerundet, um das Verletzungsrisiko für abwandernde Fische zu minimieren. In Bild 8 ist dieser Ausführungsvorschlag dargestellt.

\begin{tabular}{|l|l|l|l|l|l|l|l|}
\hline & $\mathrm{h}_{1}$ & $\mathrm{~V} \max$ & $\Delta \mathrm{P}$ & \multicolumn{2}{l|}{ Kritischer Bereich } & \multicolumn{2}{l|}{ Übergangsbereich } \\
\hline & {$[\mathrm{m}]$} & {$[\mathrm{m} / \mathrm{s}]$} & {$[\mathrm{Pa}]$} & $\mathrm{h}_{\mathrm{d}}>\mathrm{x}[\mathrm{m}]$ & $\mathrm{q}>\mathrm{y}\left[\mathrm{m}^{2} / \mathrm{s}\right]$ & $\mathrm{h}_{\mathrm{d}}>\mathrm{x}[\mathrm{m}]$ & $\mathrm{q}<\mathrm{y}\left[\mathrm{m}^{2} / \mathrm{s}\right]$ \\
\hline & & & & $\mathrm{x}$ & $\mathrm{y}$ & $\mathrm{x}$ & $\mathrm{y}$ \\
\hline Modell & 0,25 & 1,91 & 541 & 0,17 & 0,05 & 0,14 & 0,07 \\
\hline Natur & 2,0 & 5,40 & 4330 & 1,36 & 8,9 & 1,12 & 13,3 \\
\hline & 3,0 & 6,61 & 6949 & 2,04 & 24,6 & 1,68 & 36,6 \\
\hline & 4,0 & 7,64 & 8659 & 2,72 & 50,4 & 2,24 & 75,2 \\
\hline & 5,0 & 8,54 & 10824 & 3,40 & 88,0 & 2,80 & 131,4 \\
\hline
\end{tabular}

Tab. 1: $\quad$ Maßgebende Strömungsparameter für das numerische Modell und für ausgewählte Schlauchhöhen in der Natur

\section{Zusammenfassung und Ausblick}

Bei überströmten Verschlüssengibt es je nach Unterwasserstand und Abfluss Zustände, bei denen der Überfallstrahl auf die Wehrschwelle mit einem geringen Wasserpolster fällt, woraus u. U. ein erhöhtes Verletzungsrisiko für absteigende Fische entstehen kann. Die Untersuchungen zeigen, dass der Überfallstrahl bei einem Schlau chwehr mit Standard-Ablagetisch und Schlauchhöhen größer als $h_{d} / h_{1}=0,64$ auf den Ablagetisch trifft und dabei äußere und innere Verletzungen bei Fischen nicht auszuschließen sind. Hierzu ist allerdings anzumerken, dass gegenwärtig unklar ist, ab welcher Überströmungshöhe die Abwanderungsmöglichkeit von Fischen wahrgenommen wird und diese dann tatsächlich über den Verschluss absteigen.

Mit einer verkürzten Wehrschwelle kann der kritische Bereich zwar minimiert werden, allerdings hat diese Verkürzung auch Nachteile, da weniger Arbeitsraum bei der Montage und der Unterhaltung des Schlauchwehres zur Verfügung steht.

Eine weitere Reduzierung des kritischen Bereiches könnte durch eine Änderung des Wehrfahrplans erfolgen, wenn anstelle eines Parallelbetriebs die Abflusssteuerungzunächst über einen Verschluss erfolgt, bis der Unterwasserstand soweit angestiegen ist, dass ein ausreichend hohes Wasserpolster über der Wehrschwellevorliegt. Bei der Entscheidung über bauliche und betriebliche Maßnahmen 


\section{Autorenfassung}

Gebhardt, Rudolph, Kampke, Eisenhauer: Fischabstieg über Schlauchwehre: Untersuchungen der Strömungsverhältnisse und Identifizierung der Abflussbereiche mit erhöhtem Verletzungsrisiko, 2014

sollte aber zunächst die Anlagenteile einer Stauanlage identifiziert werden, die signifikant zum Fischabstieg beitragen. Eine Methode wird von Schmitt-Heiderich et al. [12] beschrieben.

Abschließend bleibtanzumerken, dass Schlauchwehre unter den gängigen Verschlusstypen im Hinblick auf die bekannten Gefährdungsfaktoren beim

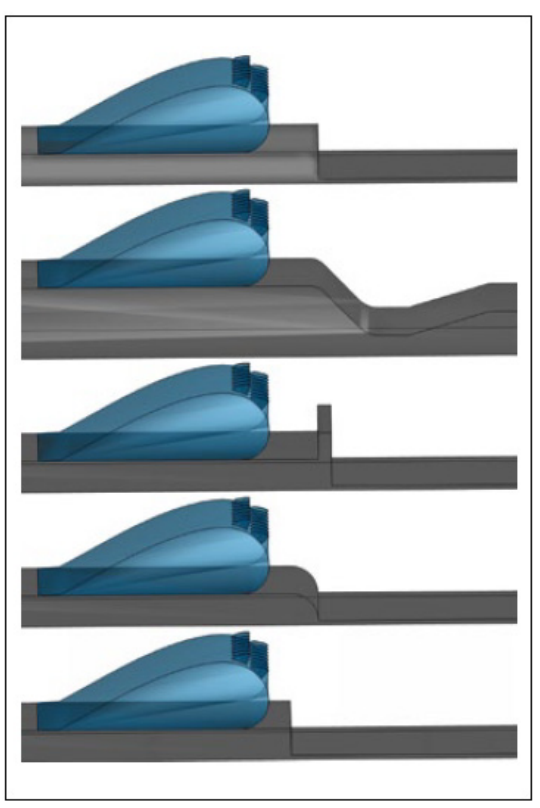

Bild 7: Untersuchte Schwellengeometrien (von oben nach unten): Standard-Wehrschwelle, ausgerundete Kante mit Muldentosbecken, Endschwelle, abgerundete Kante und verkürzte Schwelle

Gebhardt, Rudolph, Kampke, Eisenhauer: Fischabstieg über Schlauchwehre: Untersuchungen der Strömungsverhältnisse und Identifizierung der Abflussbereiche mit erhöhtem Verletzungsrisiko: Titel des Artikels. WasserWirtschaft 7/8 (2014), S. 48-53.

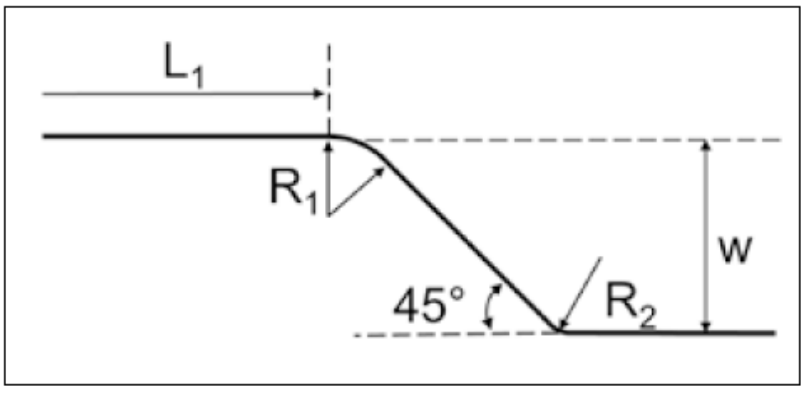

Bild 8: $\quad$ Ausführungsvorschlag für eine modifizierteWehrschwelle eines Schlauchwehrs 


\section{Autorenfassung}

Gebhardt, Rudolph, Kampke, Eisenhauer: Fischabstieg über Schlauchwehre: Untersuchungen der Strömungsverhältnisse und Identifizierung der Abflussbereiche mit erhöhtem Verletzungsrisiko, 2014

Fischabstieg grundsätzlich positiv zu bewerten sind, da dieser Verschlusstyp bei geringeren Fallhöhen bis etwa 5,0 m eingesetzt wird, woraus sich moderate Druckänderungen, geringe Aufprallgeschwindigkeiten und ein geringer Lufteintrag ergeben. Daneben besteht aufgrund der Elastizität des Verschlusses ein sehr geringes Verletzungsrisiko für die Fische. Aufgrund des Querschnitts und der gleichmäßigen Beschleunigung der Strömung stellen Schlauchwehre zudem ein geringeres Wanderhindernis dar als beispielsweise Schütze, also im hydraulischen Sinne scharfkantige Wehre [3]. Untersuchungen an einem luftgefüllten Schlauchwehr zeigen, dass die Passage über den teilweise abgesenkten Verschluss von Lachssmolts gut angenommen wurde [7].

\section{Danksagung und Hinweis}

Diese Veröffentlichung entstand mitfachlicher Unterstützung von Dr. Matthias Scholten und Dipl.Biol. Christian von Landwüst von der Bundesanstalt für Gewässerkunde (BfG) in Koblenz. Im Auftrag des Bundesministeriums für Verkehr und digitale Infrastruktur (BMVI) beraten BAW und BfG gemeinsam die Wasser- und Schifffahrtsverwaltung des Bundes bei den Maßnahmen zur Wiederherstellung der ökologischen Durchgängigkeit an Bundeswasserstraßen.

\section{Autoren}

Dr.-Ing. Michael GebhardtM. Eng. Tobias Rudolph

Dipl.-Ing. Wolfgang Kampke

Kußmaulstr. 17

76187 Karlsruhe

michael.gebhardt@baw.de

tobias.rudolph@baw.de

wolfgang.kampke@baw.de

Prof. Dr.-Ing. Norbert Eisenhauer

Hochschule Karlsruhe - Technik und Wirtschaft

Moltkestr. 30

76133 Karlsruhe

norbert.eisenhauer@hs-karlsruhe.de

\section{Literatur}

[1] Deutsche Vereinigung für Wasserwirtschaft, Abwasser und Abfall e. V. (Hrsg.): Fischschutz und Fischabstiegsanlagen. In: DWA-Themen, Hennef, 2005.

[2] Ebel, G.: Fischschutz und Fischabstieg an Wasserkraftanlagen. Handbuch Rechen- und Bypasssysteme. In: Mitteilungen aus dem Büro für Gewässerökologie und Fischereibiologie (2013), Band 4. 


\section{Autorenfassung}

Gebhardt, Rudolph, Kampke, Eisenhauer: Fischabstieg über Schlauchwehre: Untersuchungen der Strömungsverhältnisse und Identifizierung der Abflussbereiche mit erhöhtem Verletzungsrisiko, 2014

[3] Ferguson, J.W.; Poe, T. P.; Carlson, T. J.: Surface-oriented bypass systems for juvenile salmonids on the Columbia River, USA. In: Jungwirth, M., Schmutz, S.; Weiss, S. (Hrsg.): Fish migration and fish bypass. Oxford: Blackwell Scientific Publications (1998), S. 281-299.

[4] Gebhardt, M.: Hydraulische und statischeBemessung von Schlauchwehren. In: Mitteilungen des Instituts für Wasser und Gewässerentwicklung der Universität Karlsruhe (2006), Heft 235.

[5] Gebhardt, M.; Pfrommer, U.; Belzner, F., Eisenhauer, N.: 68 Jahre nach Jambor: Untersuchungen zum Einfluss einer Wehrschwelle. In: WasserWirtschaft 101 (2011), Heft 9, S. 14-19.

[6] Heisey P. G.; Mathur D.; Euston E. T.: Passing Fish Safely: A Closer Look at Turbine vs. Spillway Survival. In: Hydro Review 15 (1996), S. 42-50.

[7] Manning, D. J.; Mann, J. M.; White, K. W.; Chase, S. D.; Benkert, R. C.: Steelhead Emigration in a Seasonal Impoundment Created by an Inflatable Rubber Dam. In: North American Journal of Fisheries Management 25 (2005), S. 1239-1255.

[8] Muir, W. D.; Smith, S. G.; Williams, J. G.; Sandford, B. P.: Survival of Juvenile Salmonids Passing through Bypass Systems, Turbine and Spillways with and without Flow Deflectors at Snake River Dams. In: North American Journal of Fisheries Management 21 (2001), S. 135-146.

[9] Public Power Council Fish and Wildlife Committee (Hrsg.): Revealing Fish and Wildlife. In: Myths, 2011.

[10] Rudolph, T.: Untersuchungen zum Fischabstieg an Wehranlagen mit überströmten Wehrverschlüssen. Master-Thesisan der Hochschule Karlsruhe Wirtschaft und Technik, Bundesanstalt für Wasserbau, 2013.

[11] Ruggles, C. P.; Murray, D. G.: A review of fish response to spillways. In: Can. Techn. Report Fish. Aquat. Sciences 1172, 1983.

[12] Schmitt-Heiderich, P.; Gebhardt., M.; Weichert., R.: Klassifizierung der Wanderwege für den Fischabstieg. In: BAWBrief (2013), Nr. 2. 


\section{Autorenfassung}

Gebhardt, Rudolph, Kampke, Eisenhauer: Fischabstieg über Schlauchwehre: Untersuchungen der Strömungsverhältnisse und Identifizierung der Abflussbereiche mit erhöhtem Verletzungsrisiko, 2014

Michael Gebhardt, Tobias Rudolph, Wolfgang Kampke and Norbert Eisenhauer

Fish Passage over Inflatable Dams: Investigations of the Flow Conditions and Identification of Discharge Ranges with Increased Risk of Injury

Like almost all over-topped gate types with low tailwater levels, inflatable dams may be problematic from an ecological point of view because fish passing downstream may be injured or killed if they come in contact with the weir sill. Using numerical and hydraulic studies, the critical range of discharge is identified and the relevant hydraulic parameters are estimated. Furthermore, structural and operational measures are investigated and evaluated to reduce the risk of injury during the downstream migration of fish across inflatable dams.

Михаэль Гебхардт, Тобиас Рудольф, Вольфганг Кампке и Норберт Айзенхауер

Рыбоспуск через гибкие водосливные плотины: исследования динамики потока и идентификации областей стока с повышенным риском травмирования рыб

Гибкие водосливные плотины в местах с низким уровнем нижнего бьефа, как почти все перепускные затворы плотин, могут быть достаточно проблематичны с экологической точки зрения, так как может возникнуть опасность травмирования мигрирующих рыб на пороге затвора плотины. С помощью цифровых и гидравлических исследований эти области стока могут быть установлены, а релевантные гидравлические значения определены количественно. Кроме того, рассматриваются и оцениваются конструктивные и производственные мероприятия, сокращающие опасность ранения рыб при спуске через гибкие водосливные плотины до минимума. 